\title{
The Semimartingale Equilibrium Risk Premium for a Risk Seeking Investor
}

\author{
George M. Mukupa ${ }^{1}$, Elias R. Offen ${ }^{2}$ \\ ${ }^{1}$ School of Science, Engineering and Technology, Mulungushi University, Kabwe, Zambia \\ ${ }^{2}$ Falculty of Science, Department of Mathematics, University of Botswana, Gaborone, Botswana \\ Correspondence: George M. Mukupa, School of Science, Engineering and Technology, Mulungushi University, Kabwe, \\ Zambia
}

Received: March 29, 2020 Accepted: June 5, 2020 Online Published: July 6, 2020

doi:10.5539/jmr.v12n4p13 URL: https://doi.org/10.5539/jmr.v12n4p13

\begin{abstract}
In this paper, we consider jump amplitudes which are arbitrary and normal to study the risk seeking investor's equilibrium risk premium in the semimartingale market. We realize that, there is no optimal consumption for this investor in the market. The investor's premium differ significantly with risk aversion in both martingale and semimartingale markets in that the risk seeking investor has no optimal consumption and the wealth process only affects the rare-event premia with no effect on the diffusive premia. The compensation for this investor is highly attractive compared to risk aversion in this market.
\end{abstract}

Keywords: semimartingale, risk seeking, risk premium, jump diffusion

\section{Introduction}

The risk premium is the compensation an investor recieves for risk taking in the stock market. This premium is found by subtracting the estimated bond return from the estimated stock return. Infact, the size of the premium varies as the risk in a particular stock or in the stock market at large. It is generally true that high risk investments are compensated with a higher equity premium and vice-versa.

The reason behind this premium is to entice investors to engage themselves in riskier investments because of the higher rate of return attached to them (Simon \& John, 2005). However, an investment in stocks is less guaranteed as companies usually suffer downturns or go out of business. Infact, Aswath (2012) observes that this premium reflects fundamental judgments investors make about how much risk one sees in the market and what price one attaches to that risk. In this regard, investors can base their payment for a share of stock on the risk perceived and anticipated stock return. This will enable them calibrate their investments in a manner that properly compensates them for the excess risk they are taking.

If the consumer has reasonable preferences then it is possible to use utility function to describe these preferences (Bellamy \& Jeanblanc, 2000). As (Eberlein \& Jacod, 1997) states, utility (happiness) depends on consumption. The more the consumption, the more the utility. However, the increase in utility which results from the increase in consumption is smaller the more consumption you already have. This implies that utility is a concave function (Mukupa \& Offen, 2015). This follows directly from the fact that people do not like risks and so to induce people to substitute to a risky alternative, it is vital to compensate them by making the riskless alternative unfair and that is by giving less than its expected value. This makes investors indifferent between undertaking the risky or non risky investment.The certainty equivalent (C) for some investment whose outcome is a random variable $\mathrm{Z}$ is

$$
U(C)=E[U(Z)]
$$

In this paper, the investor with utility function $\mathrm{U}$ has current wealth less than $\mathrm{C}$ so that the investment is attractive.

It is important to consider that much of the work in finance has been based on martingale markets whose future is deemed fair and unpredictable by normalizing prices. This gives investors a fair chance to either gain or lose out on their investments. In this paper, we consider a market $X_{t}$ to allow a decomposition

$$
X_{t}=X_{0}+M+A \text {, }
$$

such that $M=\left(M_{t}\right)_{0 \leq t \leq T}$ is a square-integrable martingale with $M_{0}=0$ and $A=\left(A_{t}\right)_{0 \leq t \leq T}$ is a predictable process of finite variation $|A|$ with $A_{0}=0$. 
Here, the semi martingale approach is used to determine equilibrium equity premium for a risk seeking investor in a production economy with jumps as opposed to option pricing. This is because several articles [(Bellamy \& Jeanblanc, 2000), (El Karoui, 1998), (Hobson, 1998), (Henderson, 2005), (Henderson, 2003), (Henderson \& Hobson, 2003), (Moller, 2004), (Jakubenas, 2002), (Gushchin \& Mordecki, 2002), (Frey \& Sin, 1999)] have addressed the option pricing problem. The results for models with nontrivial pricing intervals and the corresponding comparison results are less complete. (Henderson \& Hobson, 2003) proved that the price of a European call for a diffusion with jumps is bounded below by the corresponding Black-Scholes price and above by the trivial upper price (see also (Hobson, 2005) and (Frontczak, 2013) for alternative proofs). An important generalization of the technique introduced in (Bellamy \& Jeanblanc, 2000) and (El Karoui, 1998) has been established by (Gushchin \& Mordecki, 2002) who derive a general comparison result for one-dimensional semimartingales.

In this paper, we take the certainty equivalent to be greater than the expected profit for the alternative so that the investor is risk seeking or risk loving. Therefore, the graph of utility is convex. We can then compare our paper to (Zhang, Zhao \& Chang, 2012) and also further elaboration by (Mukupa \& Offen 2015), (Mukupa \& Offen, 2016), (Mukupa, Offen, Kunda \& Lungu, 2016), (Mukupa, Offen \& Lungu, 2016), (Mukupa \& Offen, 2018a) and (Mukupa \& Offen, 2018b) although they considered the case for risk averse investors.

\section{The Model}

Our price process evolves according to the stochastic differential equation;

$$
d X_{t}=\mu d t+\delta d B_{t}+\left(e^{x}-1\right) d N_{t}-\lambda E\left(e^{x}-1\right) d t .
$$

which is a semimartingale with discontinuities because of the presence of jumps.

We take $\mu, \delta$ and $\lambda$ as constants and $x$ as a vector of arbitrary distributed jump sizes. Thereafter, we fix the amplitudes to be normally distributed.The processes $B_{t}$ and $N_{t}$ are independent. This follows directly from the definition of Brownian motion as being a continuous process and the Poisson being discrete which we obviously know that continuous processes and discrete are independent. $\lambda$ is the frequency of the Poisson process. We set $\left(e^{x}-1\right)$ in the jump process so that $e^{x}-1=0$ if there is no jump as $x$ is then a zero vector. $E$ is the expectation which makes the process $e^{x}-1$ deterministic. $d N_{t}$ models the sudden changes as a result of rare events happening and $d B_{t}$ models small continuous changes generated by the noise whose volatility is a constant $\delta$.

The compensated compound Poisson process $\left(e^{x}-1\right) d N_{t}-\lambda E\left(e^{x}-1\right) d t$ has the mean of zero because

$$
E\left[\left(e^{x}-1\right) d N_{t}-\lambda E\left(e^{x}-1\right) d t\right]=E\left(e^{x}-1\right) E\left(d N_{t}\right)-E\left(e^{x}-1\right) E(\lambda d t)=0
$$

and $E\left(d N_{t}\right)=\lambda d t$.

To solve

$$
d X_{t}=\mu d t+\delta d B_{t}+\left(e^{x}-1\right) d N_{t}-\lambda E\left(e^{x}-1\right) d t,
$$

we do not need to apply Itô Lemma with Jumps because the diffusion part is a continuous semi martingale whose procedure for solution does not require the integrating factor. We solve for the price process at the terminal time $T$ as follows;

$$
d X_{t}=\left[\mu-\lambda E\left(e^{x}-1\right)\right] d t+\delta d B_{t}+\left(e^{x}-1\right) d N_{t}
$$

By integration we have

$$
X_{T}=X_{t}+\left[\mu-\lambda E\left(e^{x}-1\right)\right] \tau+\delta B_{\tau}+\sum_{i=1}^{N_{\tau}}\left(e^{x_{i}}-1\right), \text { for } \tau=T-t
$$

as the investment period.

Suppose also that, at the risk-free rate $\rho$, the money market account $X_{0}(t)$ is such that

$$
d X_{0}(t)=\rho(t) X_{0}(t) d t
$$

whose total supply is assumed to be zero. Consider here that $\rho$ is risk-free because it is the rate for the non risky asset (money account).

Since the value of someone's investment in this production economy at any time $t$ is given by $V_{t}=\phi X_{t}$, for some portfolio $\phi=(1-\omega, \omega)$ consisting of $1-\omega$ non risky assets and $\omega$ risky assets, we have that by the self financing strategy,

$$
d V_{t}=\phi d X_{t}
$$


so that the total wealth at any time $t$ is

$$
V_{t}=V_{0}(t)+V_{1}(t)
$$

where $V_{0}(t)$ is the value of the money market account and $V_{1}(t)$ is the value of the investment in the stock market at time $t$.

Now

$$
\begin{gathered}
d V_{t}=d V_{0}(t)+d V_{1}(t) \\
=(1-\omega) d X_{0}(t)+\omega d X(t) \\
=(1-\omega)\left(\rho X_{0}(t) d t\right)+\omega\left[\mu-\lambda E\left(e^{x}-1\right)\right] d t+\omega \delta d B_{t}+\omega\left(e^{x}-1\right) d N_{t} .
\end{gathered}
$$

Since the equity premium $\phi=\mu-\rho$, we have that $\mu=\phi+\rho$, hence

$$
d V_{t}=\left[\rho X_{0}(t)-\omega \rho X_{0}(t)+\omega \phi+\omega \rho-\lambda \omega E\left(e^{x}-1\right)\right] d t+\omega \delta d B_{t}+\omega\left(e^{x}-1\right) d N_{t} .
$$

The investor's optimal control problem then is to maximize his expected utility function

$$
\max E_{t} \int_{t}^{T} y(t) U\left(r_{t}\right) d t
$$

subject to

$$
d V_{t}=\left[\rho X_{0}(t)-\omega \rho X_{0}(t)+\omega \phi+\omega \rho-\lambda \omega E\left(e^{x}-1\right)-r_{t}\right] d t+\omega \delta d B_{t}+\omega\left(e^{x}-1\right) d N_{t}
$$

The wealth ratio $\omega$ and consumption rate $r_{t}$ are control variable. The general equilibrium occur when $\omega=1$.

\section{Results and Discussions}

Theorem 1 In a semimartingale market with arbitrary jumps, an investor's equilibrium risk premium with the risk seeking utility function $U\left(r_{t}\right)=p^{-r_{t}}, 0<p<1$ in the production economy is given by

$$
\phi=\rho X_{0}(t)-\rho+\lambda E\left(e^{x}-1\right)+\sigma^{2} \ln p-\frac{\lambda}{Q_{t} p^{-V_{t}} \ln p} E\left[Q_{t} p^{-V_{t} e^{x}}(\ln p) V_{t}\left(e^{x}-1\right)\right] .
$$

where $\phi_{\delta}=\rho X_{0}(t)-\rho+\sigma^{2} \ln p$ is the diffusive risk premium and $\phi_{N}=\lambda E\left(e^{x}-1\right)-\frac{\lambda}{Q_{t} p^{-V_{t}} \ln p} E\left[Q_{t} p^{-V_{t} e^{x}}(\ln p) V_{t}\left(e^{x}-1\right)\right]$ is the rare-event premium. We realize here that, the diffusive risk always exist even if we normalize the market. Also, the wealth process only affects the rare-event premia.

Proof. We optimize the investor's utility based on the Hamilton-Jacobi-Bellman (HJB) equation

$$
E_{t}\left[d J+y U\left(r_{t}\right) d t\right]=0 .
$$

Now,

$$
d V_{t}=\left[\rho X_{0}(t)-\omega \rho X_{0}(t)+\omega \phi+\omega \rho-\omega \lambda E\left(e^{x}-1\right)-r_{t}\right] d t+\omega \delta d B_{t}+\omega\left(e^{x}-1\right) d N_{t}
$$

Take $d^{*} V_{t}$ as the diffusion part;

$$
d^{*} V_{t}=\left[\rho X_{0}(t)-\omega \rho X_{0}(t)+\omega \phi+\omega \rho-\omega \lambda E\left(e^{x}-1\right)-r_{t}\right] d t+\omega \delta d B_{t}
$$

then

$$
d^{*} J=J_{t} d t+J_{V_{t}} d^{*} V_{t}+\frac{1}{2} J_{V_{t} V_{t}}\left(d^{*} V_{t}\right)^{2}
$$

so that

$$
d J=d^{*} J+\left[J\left(V_{t}\left(1+\omega\left(e^{x}-1\right)\right), t\right)-J\left(V_{t}, t\right)\right] d N_{t}
$$




$$
\begin{gathered}
=J_{t} d t+J_{V_{t}} d^{*} V_{t}+\frac{1}{2} J_{V_{t} V_{t}}\left(d^{*} V_{t}\right)^{2}+\left[J\left(V_{t}\left(1+\omega\left(e^{x}-1\right)\right), t\right)-J\left(V_{t}, t\right)\right] d N_{t} \\
\left.=J_{t} d t+J_{V_{t}}\left[\rho \rho X_{0}(t)-\omega \rho X_{0}(t)+\omega \phi+\omega \rho-\omega \lambda E\left(e^{x}-1\right)-r_{t}\right] d t+\omega \delta d B_{t}\right]+\frac{1}{2} J_{V_{t} V_{t}}\left[\omega^{2} \delta^{2} d t\right] \\
+\left[J\left(V_{t}\left(1+\omega\left(e^{x}-1\right)\right), t\right)-J\left(V_{t}, t\right)\right] d N_{t} \\
d J=J_{t} d t+J_{V_{t}}\left[\rho X_{0}(t)-\omega \rho X_{0}(t)+\omega \phi+\omega \rho-\omega \lambda E\left(e^{x}-1\right)-r_{t}\right] d t+J_{V_{t}} \omega \delta d B_{t}+\frac{1}{2} J_{V_{t} V_{t}}\left[\omega^{2} \delta^{2} d t\right] \\
+\left[J\left(V_{t}\left(1+\omega\left(e^{x}-1\right)\right), t\right)-J\left(V_{t}, t\right)\right] d N_{t} .
\end{gathered}
$$

Take

$$
E_{t}\left[d J+y U\left(r_{t}\right) d t\right]=0
$$

$$
\begin{gathered}
J_{t} d t+J_{V_{t}}\left[\rho X_{0}(t)-\omega \rho X_{0}(t)+\omega \phi+\omega \rho-\omega \lambda E\left(e^{x}-1\right)-r_{t}\right] d t+\frac{1}{2} J_{V_{t} V_{t}} \omega^{2} \delta^{2} d t \\
+E\left[J\left(V_{t}\left(1+\omega\left(e^{x}-1\right)\right), t\right)-J\left(V_{t}, t\right)\right] \lambda d t+y U\left(r_{t}\right) d t=0
\end{gathered}
$$

divide through by $\mathrm{dt}$

$$
\begin{gathered}
J_{t}+J_{V_{t}}\left[\rho X_{0}(t)-\omega \rho X_{0}(t)+\omega \phi+\omega \rho-\omega \lambda E\left(e^{x}-1\right)-r_{t}\right]+\frac{1}{2} J_{V_{t} V_{t}} \omega^{2} \delta^{2} \\
+\lambda E\left[J\left(V_{t}\left(1+\omega\left(e^{x}-1\right)\right), t\right)-J\left(V_{t}, t\right)\right]+y U\left(r_{t}\right)=0
\end{gathered}
$$

To evaluate

$$
\begin{gathered}
\max _{\left(r_{t}, \omega\right)}\left\{J_{t}+J_{V_{t}}\left[\rho X_{0}(t)-\omega \rho X_{0}(t)+\omega \phi+\omega \rho-\omega \lambda E\left(e^{x}-1\right)-r_{t}\right]+\frac{1}{2} J_{V_{t} V_{t}} \omega^{2} \delta^{2}\right. \\
\left.+\lambda E\left[J\left(V_{t}\left(1+\omega\left(e^{x}-1\right)\right), t\right)-J\left(V_{t}, t\right)\right]+y U\left(r_{t}\right)=0\right\},
\end{gathered}
$$

take partial derivatives with respect to $r_{t}$ and $\omega$ to obtain the first order conditions

$$
\begin{gathered}
-J_{V_{t}}+y U\left(r_{t}\right)=0, \\
{\left[-\rho X_{0}(t)+\phi+\rho-\lambda E\left(e^{x}-1\right)\right] J_{V_{t}}+J_{V_{t} V_{t}} \omega \delta^{2}+\lambda E\left[J_{V_{t}}\left(V_{t}\left(1+\omega\left(e^{x}-1\right)\right), t\right) V_{t}\left(e^{x}-1\right)\right]=0 .}
\end{gathered}
$$

Solving for $\phi$ in the second equation and taking the equilibrium condition $\omega=1$ yields the general equilibrium equity premium

$$
\phi=\rho X_{0}(t)-\rho+\lambda E\left(e^{x}-1\right)-\frac{J_{V_{t} V_{t}}}{J_{V_{t}}} \delta^{2}-\frac{\lambda}{J_{V_{t}}} E\left[J_{V_{t}}\left(V_{t} e^{x}, t\right) V_{t}\left(e^{x}-1\right)\right] .
$$

Substituting this $\phi$ into the Bellman equation

$$
\begin{gathered}
J_{t}+J_{V_{t}} \rho X_{0}(t)-J_{V_{t}} \rho X_{0}(t)+J_{V_{t}}\left[\rho X_{0}(t)-\rho+\lambda E\left(e^{x}-1\right)-\frac{J_{V_{t} V_{t}}}{J_{V_{t}}} \delta^{2}-\frac{\lambda}{J_{V_{t}}} E\left[J_{V_{t}}\left(V_{t} e^{x}, t\right) V_{t}\left(e^{x}-1\right)\right]\right. \\
\left.+J_{V_{t}} \rho-J_{V_{t}} \lambda E\left(e^{x}-1\right)-J_{V_{t}} r_{t}+\frac{1}{2} J_{V_{t} V_{t}} \delta^{2}+\lambda E\left[J\left(V_{t} e^{x}\right), t\right)-J\left(V_{t}, t\right)\right]+y U\left(r_{t}\right)=0
\end{gathered}
$$

which simplifies to the integro p.d.e

$$
\begin{gathered}
\left.J_{t}+J_{V_{t}} \rho X_{0}(t)-J_{V_{t}} r_{t}-\frac{1}{2} J_{V_{t} V_{t}} \delta^{2}-\lambda E\left[J_{V_{t}}\left(V_{t} e^{x}, t\right) V_{t}\left(e^{x}-1\right)\right]+\lambda E\left[J\left(V_{t} e^{x}\right), t\right)-J\left(V_{t}, t\right)\right] \\
+y U\left(r_{t}\right)=0
\end{gathered}
$$


Consider now the risk seeking utility function

$$
U\left(r_{t}\right)=p^{-r_{t}}, \quad p<1,
$$

which is a convex function. We solve for $J\left(V_{t}, t\right)$ based on the indirect utility

$$
J\left(V_{t}, t\right)=Q(t) p^{-V_{t}} .
$$

The optimal consumption will be solved from the first order conditiion (0.1) as:

$$
y U^{\prime}\left(r_{t}\right)=J_{V_{t}}
$$

which by implicit differentiation yields

$$
U^{\prime}\left(r_{t}\right)=-p^{-r_{t}} \ln p
$$

and hence

$$
y\left[-p^{-r_{t}} \ln p\right]=Q(t) V_{t}^{\beta-1}
$$

and therefore

$$
r_{t}=\frac{-\ln \left(\frac{-Q_{t} V_{t}^{\beta-1}}{y \ln p}\right)}{\ln p}
$$

meaning that the optimal consumption we require does not exist because the log of a negative number does not exist. This is because $Q_{t}$ and $V_{t}$, are functions of time hence they can not yield a negative result. Also $0<p<1$.

Substituting the functions $J\left(V_{t}, t\right)=Q(t) p^{-V_{t}}, J_{V_{t}}=-Q(t) p^{-V_{t}} \ln p$ and $J_{V_{t} V_{t}}=Q(t) p^{-V_{t}}(\ln p)^{2}$ into the integro p.d.e gives

$$
\begin{gathered}
Q_{t} p^{-V_{t}}-Q_{t} p^{-V_{t}}(\ln p) \rho X_{0}(t)+r_{t} Q_{t} p^{-V_{t}} \ln p-0.5 \sigma^{2} Q_{t} p^{-V_{t}}(\ln p)^{2} \\
-\lambda E\left[-Q_{t} p^{-V_{t} e^{x}}(\ln p) V_{t}\left(e^{x}-1\right)\right]+\lambda E\left[Q_{t} p^{-V_{t} e^{x}}-Q_{t} p^{-V_{t}}\right]+y p^{-r_{t}}=0
\end{gathered}
$$

differentiating with respect to $V_{t}$ and dividing through by $-(\ln p) p^{-V_{t}}$ gives the terminal conditions

$$
\begin{gathered}
Q_{t}-Q_{t}(\ln p) \rho X_{0}(t)+Q_{t} r_{t}(\ln p)+0.5 \sigma^{2} Q_{t}(\ln p)^{2} \\
+\lambda E\left[V_{t}\left(e^{x}-1\right) e^{x}(\ln p) Q_{t} p^{-V_{t}\left(e^{x}-1\right)}-Q_{t} p^{-V_{t}\left(e^{x}-1\right)}\left(e^{x}-1\right)\right]-\lambda E\left[Q_{t}-e^{x} Q_{t} p^{-V_{t}\left(e^{x}-1\right)}\right]=0
\end{gathered}
$$

and

$$
Q(T)=0,
$$

Substituting $J\left(V_{t}, t\right)=Q(t) p^{-V_{t}}, J_{V_{t}}=-Q(t) p^{-V_{t}} \ln p$ and $J_{V_{t} V_{t}}=Q(t) p^{-V_{t}}(\ln p)^{2}$ into the general equilibrium equity premium gives us the risk premium for arbitrary jumps as;

$$
\phi=\rho X_{0}(t)-\rho+\lambda E\left(e^{x}-1\right)+\sigma^{2} \ln p-\frac{\lambda}{Q_{t} p^{-V_{t}} \ln p} E\left[Q_{t} p^{-V_{t} e^{x}}(\ln p) V_{t}\left(e^{x}-1\right)\right] .
$$

Notice here that, if we normalize the market by $X_{0}(t)=1$, the equilibrium equity premium will be given by

$$
\phi=\lambda E\left(e^{x}-1\right)+\sigma^{2} \ln p-\frac{\lambda}{Q_{t} p^{-V_{t}} \ln p} E\left[Q_{t} p^{-V_{t} e^{x}}(\ln p) V_{t}\left(e^{x}-1\right)\right] .
$$

This is not consistent to taking $\rho=0$ in martingale markets. We also observe that the value process affects only the rare-event premia but it has no effect on the diffusive premia.

Theorem 2 When jump amplitudes are normally distributed, the risk seeking investor's risk premium in the semi martingale market is given by

$$
\left.\phi=\rho X_{0}(t)-\rho+\sigma^{2} \ln p+\lambda\left(e^{\mu_{x}+0.5 \sigma_{x}^{2}}\right)-\lambda-\lambda V_{t}\left(e^{\mu_{x}+0.5 \sigma_{x}^{2}}-1\right) p^{-V_{t}\left(1-e^{\mu_{x}+0.5 \sigma_{x}^{2}}\right)}\right]
$$


Proof. Since $X \sim N\left(\mu_{x}, \sigma_{x}^{2}\right)$ then $e^{X}$ is a lognormal random variable with parameters $\mu_{x}$ and $\sigma_{x}^{2}$ thus

$$
E\left[e^{X}\right]=E\left[e^{x}\right]=e^{\mu_{x}+\frac{1}{2} \sigma_{x}^{2}} .
$$

For the risk seeking utility function, the rare-event premium

$$
\phi_{N}=\lambda E\left(e^{x}-1\right)-\frac{\lambda}{Q_{t} p^{-V_{t}} \ln p} E\left[Q_{t} p^{-V_{t} e^{x}}(\ln p) V_{t}\left(e^{x}-1\right)\right]
$$

Now

$$
\begin{gathered}
\lambda E\left(e^{x}\right)-\lambda-\frac{\lambda Q_{t} V_{t} \ln p}{Q_{t} p^{-V_{t}} \ln p} E\left[p^{-V_{t} e^{x}}\left(e^{x}-1\right)\right] \\
\lambda E\left(e^{x}\right)-\lambda-\frac{\lambda V_{t}}{p^{-V_{t}}} E\left[p^{-V_{t} e^{x}}\left(e^{x}-1\right)\right] \\
\lambda E\left(e^{x}\right)-\lambda-\frac{\lambda V_{t}}{p^{-V_{t}}}\left[E\left(p^{-V_{t} e^{x}} e^{x}\right)-E\left(p^{-V_{t} e^{x}}\right)\right]
\end{gathered}
$$

Now, since $X \sim N\left(\mu_{x}, \sigma_{x}^{2}\right)$ but $V_{t}$ is not normally distributed, the two processes are uncorrelated and hence

$$
\begin{gathered}
\lambda\left(e^{\mu_{x}+0.5 \sigma_{x}^{2}}\right)-\lambda-\frac{\lambda V_{t}}{p^{-V_{t}}}\left[e^{\mu_{x}+0.5 \sigma_{x}^{2}} p^{-V_{t}\left(e^{\mu x+0.5 \sigma_{x}^{2}}\right)}-p^{-V_{t}\left(e^{\mu_{x}+0.5 \sigma_{x}^{2}}\right)}\right] \\
\lambda\left(e^{\mu_{x}+0.5 \sigma_{x}^{2}}\right)-\lambda-\frac{\lambda V_{t}}{p^{-V_{t}}}\left[p^{-V_{t}\left(e^{\mu_{x}+0.5 \sigma_{x}^{2}}\right)}\left(e^{\mu_{x}+0.5 \sigma_{x}^{2}}-1\right)\right]
\end{gathered}
$$

Therefore, the equilibrium equity premium is:

$$
\left.\phi=\rho X_{0}(t)-\rho+\sigma^{2} \ln p+\lambda\left(e^{\mu_{x}+0.5 \sigma_{x}^{2}}\right)-\lambda-\lambda V_{t}\left(e^{\mu_{x}+0.5 \sigma_{x}^{2}}-1\right) p^{-V_{t}\left(1-e^{\mu_{x}+0.5 \sigma_{x}^{2}}\right)}\right]
$$

We realise in Figure 1 that the equity premium is almost zero whenever volatility is zero. This result is similar to the risk averse investor in this market. It can be concluded from this result that the deterministic process yields no significant equilibrium equity premium although the premium realised here is better than that of the risk averse investor. This is an important result because the premium is only dependent on the volatility in the diffusive risk and not in the jump risk. The premium is also symmetrical about zero volatility. In Figure 2 and Figure 3, we see that the equity premium increases with an increase in the mean or variance. In this regard, it is highly dependent on the mean and variance of the normality in the jump sizes. In Figure 4, the premium decreases with an increase in the wealth process. This is because, the more the wealth of this investor, the less is the exposure to the jump risk. 


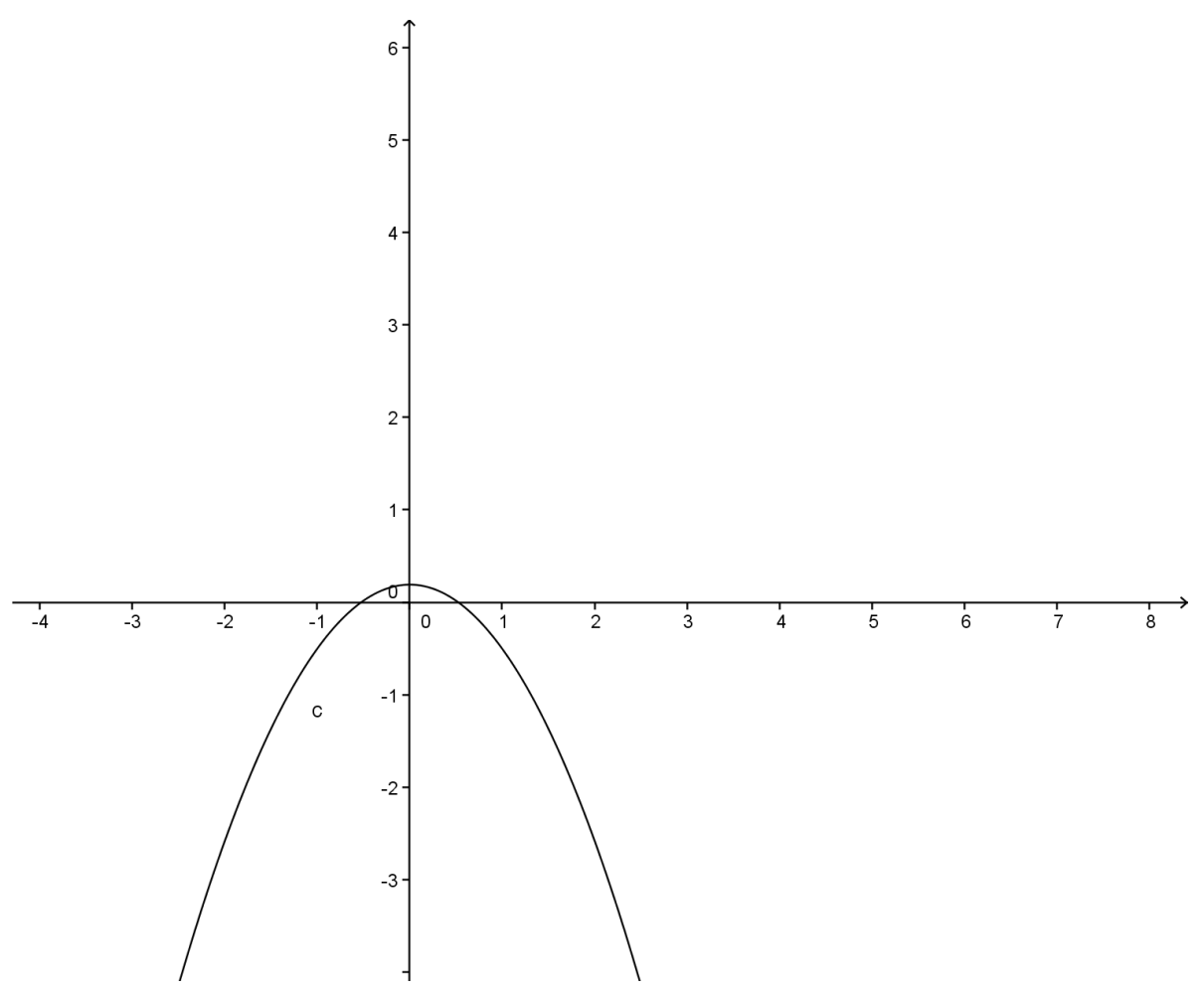

Figure 1. Volatility Effect on Equity Premium

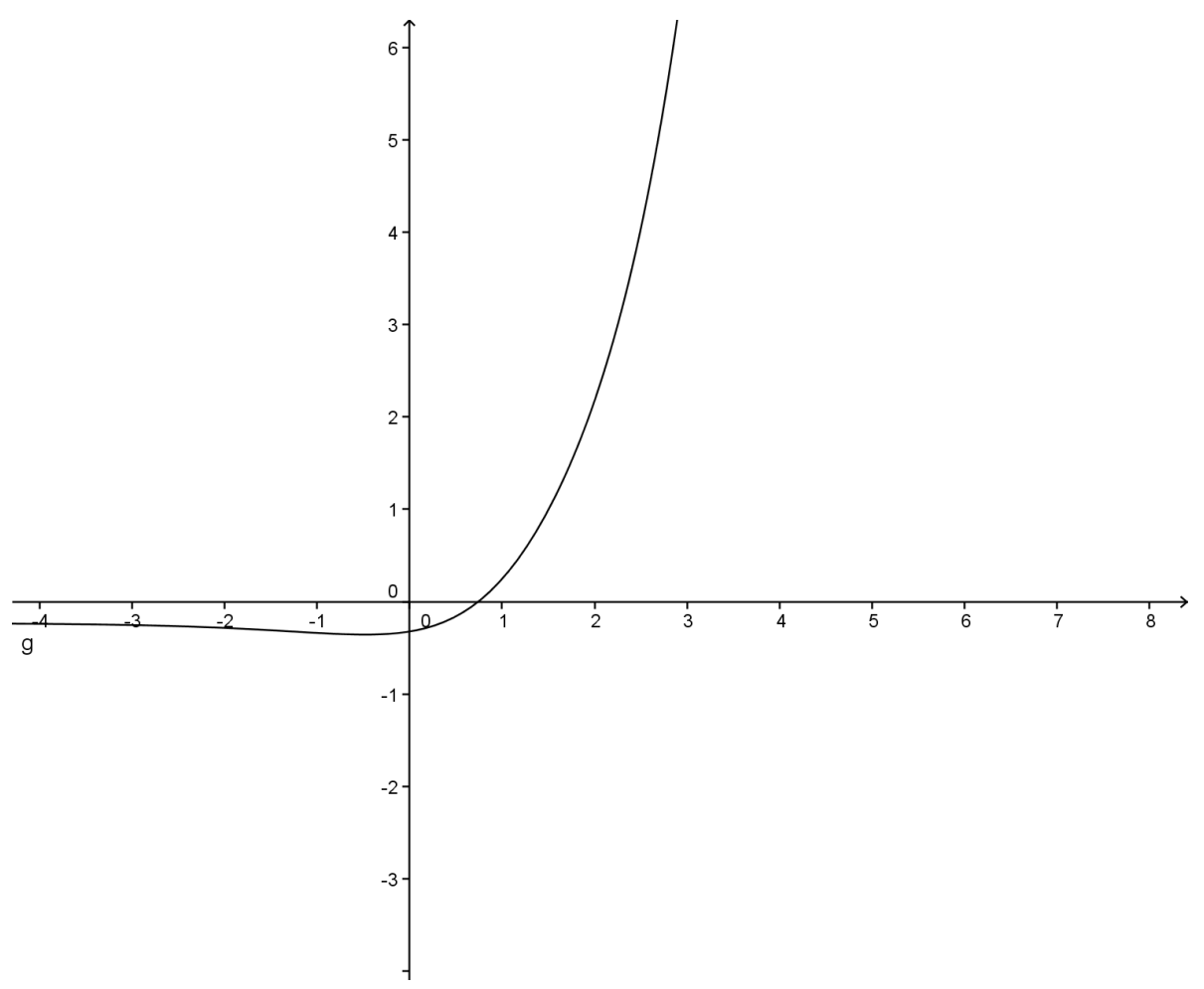

Figure 2. Mean Effect on Equity Premium 


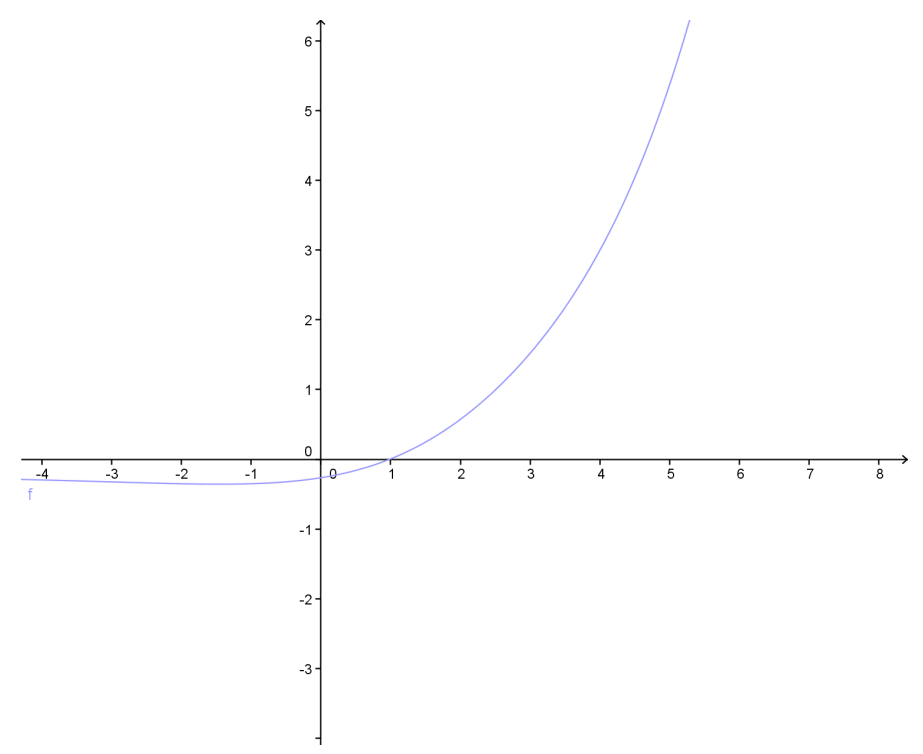

Figure 3. Variance Effect on Equity Premium

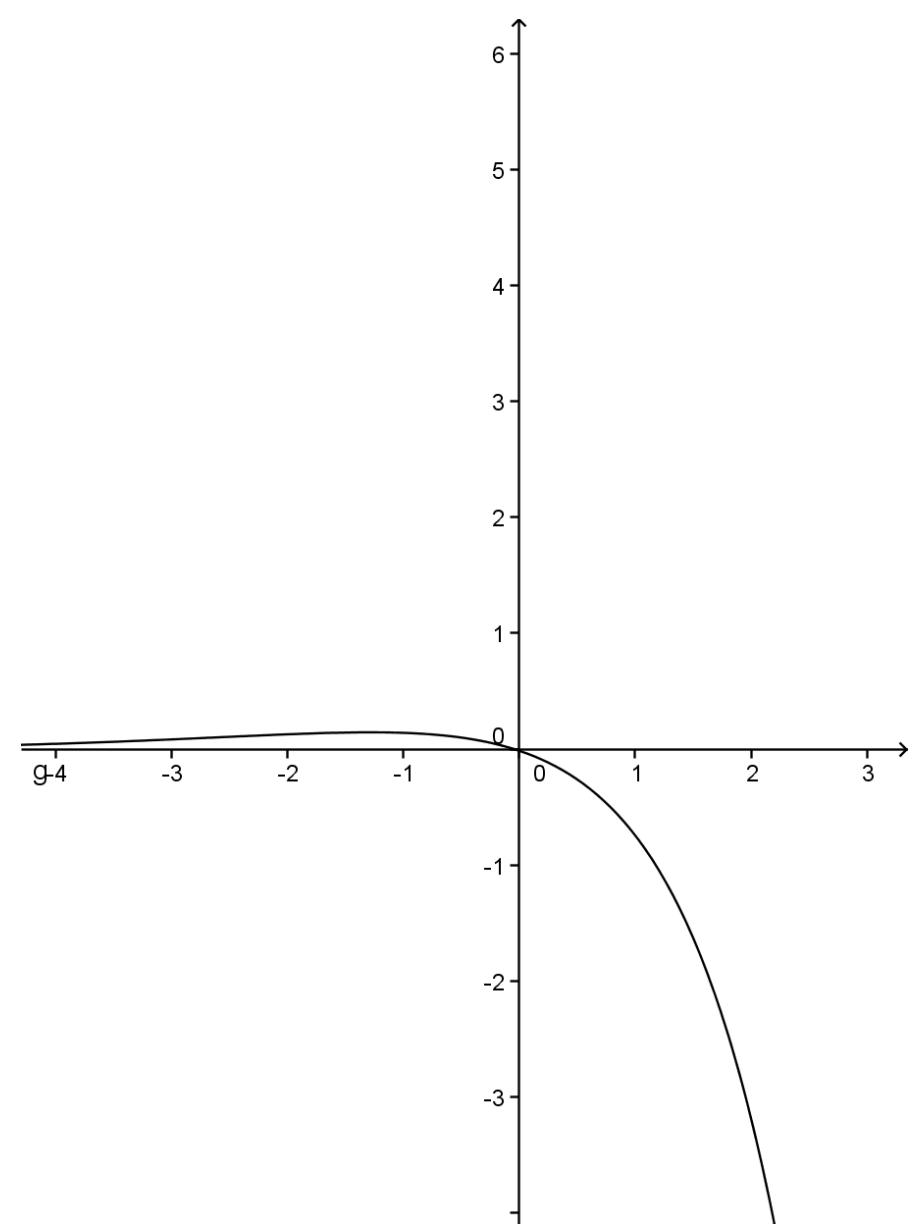

Figure 4. Wealth Effect on Equity Premium

Theorem 3 In the semimartingale market, the investor's equilibrium equity premium in the production economy with normally distributed jumps under the CARA negative exponential utility function $U\left(r_{t}\right)=-e^{-\alpha r_{t}}, \alpha>0$, is given by

$$
\phi=\rho X_{0}(t)-\rho+\alpha \delta^{2}+\lambda e^{\mu_{x}+\frac{1}{2} \sigma_{x}^{2}}-\lambda q \exp \left[\mu_{x}+q \alpha-q \alpha e^{\mu_{x}+\frac{1}{2} \sigma_{x}^{2}}\right]
$$




$$
\begin{gathered}
{\left[\frac{\exp \left\{\frac{1}{2}\left[\sigma_{x}^{2}+\mu_{x}^{2}+2 \alpha q \mu_{x}-2 \alpha q \mu_{x} e^{\mu_{x}+\frac{1}{2} \sigma_{x}^{2}}+\alpha^{2} q^{2}-2 \alpha^{2} q^{2} e^{\mu_{x}+\frac{1}{2} \sigma_{x}^{2}}+\alpha^{2} q^{2} e^{2 \mu_{x}+2 \sigma_{x}^{2}}\right]\right\}}{\exp \left\{\frac{1}{2}\left[\mu_{x}+\alpha q-\alpha q e^{\mu_{x}+\frac{1}{2} \sigma_{x}^{2}}\right]^{2}\right\}}\right]} \\
-\lambda+\lambda q e^{\alpha q-\alpha q e^{\mu_{x}+\frac{1}{2} \sigma_{x}^{2}}+\frac{1}{2}\left[\alpha^{2} q^{2}-2 \alpha^{2} q^{2} e^{\mu_{x}+\frac{1}{2} \sigma_{x}^{2}}+\alpha^{2} q^{2} e^{2 \mu_{x}+2 \sigma_{x}^{2}}-\left[\alpha q-\alpha q e^{\mu_{x}+\frac{1}{2} \sigma_{x}^{2}}\right]^{2}\right]}
\end{gathered}
$$

Proof. For a CARA Negative exponential utility function, the rare-event premium

$$
\begin{aligned}
\phi_{N} & =\lambda E\left[\left(e^{x}-1\right)\left(1-\frac{V_{t} e^{-\alpha V_{t} e^{x}}}{e^{-\alpha V_{t}}}\right)\right] \\
& =\lambda E\left[e^{x}-e^{x+\alpha V_{t}} \cdot V_{t} e^{-\alpha V_{t} e^{x}}-1+\frac{V_{t} e^{-\alpha V_{t} e^{x}}}{e^{-\alpha V_{t}}}\right] \\
& =\lambda E\left[e^{x}-V_{t} e^{x+\alpha V_{t}\left(1-e^{x}\right)}-1+V_{t} e^{\alpha V_{t}\left(1-e^{x}\right)}\right] \\
& =\lambda\left[E\left(e^{x}\right)-q E\left(e^{x+\alpha V_{t}\left(1-e^{x}\right)}\right)-1+q E\left(e^{\alpha V_{t}\left(1-e^{x}\right)}\right)\right]
\end{aligned}
$$

Now $X \sim N\left(\mu_{x}, \sigma_{x}^{2}\right)$,

$$
\begin{aligned}
E\left[x+\alpha V_{t}\left(1-e^{x}\right)\right] & =E[x]+E\left[\alpha V_{t}\left(1-e^{x}\right)\right] \\
& =E[x]+\alpha q-\alpha q E\left[e^{x}\right] \\
& =\mu_{x}+\alpha q-\alpha q e^{\mu_{x}+\frac{1}{2} \sigma_{x}^{2}}
\end{aligned}
$$

Also

$$
\begin{gathered}
\operatorname{var}\left[x+\alpha V_{t}\left(1-e^{x}\right)\right]=E\left[\left(x+\alpha V_{t}\left(1-e^{x}\right)\right)^{2}\right]-\left[E\left[x+\alpha V_{t}\left(1-e^{x}\right)\right]\right]^{2} \\
\operatorname{var}\left[x+\alpha V_{t}\left(1-e^{x}\right)\right]=E\left[x^{2}+2 x V_{t} \alpha\left(1-e^{x}\right)+\alpha^{2} V_{t}^{2}\left(1-e^{x}\right)^{2}\right]-\left[\mu_{x}+\alpha q-\alpha q e^{\mu_{x}+\frac{1}{2} \sigma_{x}^{2}}\right]^{2} \\
=E\left[x^{2}\right]+2 q \alpha E[x]-2 q \alpha E[x] E\left[e^{x}\right]+\alpha^{2} q^{2} E\left(1-2 e^{x}+e^{2 x}\right)-\left[\mu_{x}+\alpha q-\alpha q e^{\mu_{x}+\frac{1}{2} \sigma_{x}^{2}}\right]^{2} \\
=\sigma_{x}^{2}+\mu_{x}^{2}+2 q \alpha \mu_{x}-2 q \alpha \mu_{x} e^{\mu_{x}+\frac{1}{2} \sigma_{x}^{2}}+\alpha^{2} q^{2}\left(1-2 e^{\mu_{x}+\frac{1}{2} \sigma_{x}^{2}}+e^{2 \mu_{x}+2 \sigma_{x}^{2}}\right)-\left[\mu_{x}+\alpha q-\alpha q e^{\mu_{x}+\frac{1}{2} \sigma_{x}^{2}}\right]^{2}
\end{gathered}
$$

Thus $E\left[e^{x+\alpha V_{t}\left(1-e^{x}\right)}\right]$ is then,

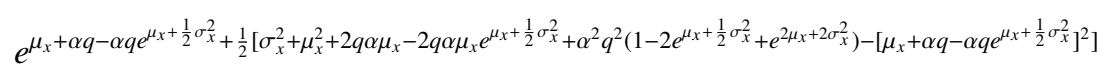

Also

$$
E\left[\alpha V_{t}\left(1-e^{x}\right)\right]=\alpha q-\alpha q e^{\mu_{x}+\frac{1}{2} \sigma_{x}^{2}}
$$

and

$$
\begin{aligned}
& \operatorname{var}\left[\alpha V_{t}\left(1-e^{x}\right)\right]=E\left[\left(\alpha V_{t}\left(1-e^{x}\right)\right)^{2}\right]-\left[\alpha q-\alpha q e^{\mu_{x}+\frac{1}{2} \sigma_{x}^{2}}\right]^{2} \\
& =\alpha^{2} q^{2} E\left(1-e^{x}\right)^{2}-\left[\alpha q-\alpha q e^{\mu_{x}+\frac{1}{2} \sigma_{x}^{2}}\right]^{2} \\
& =\alpha^{2} q^{2} E\left(1-2 e^{x}+e^{2 x}\right)-\left[\alpha q-\alpha q e^{\mu_{x}+\frac{1}{2} \sigma_{x}^{2}}\right]^{2} \\
& =\alpha^{2} q^{2}\left(1-2 e^{\mu_{x}+\frac{1}{2} \sigma_{x}^{2}}+e^{2 \mu_{x}+2 \sigma_{x}^{2}}\right)-\left[\alpha q-\alpha q e^{\mu_{x}+\frac{1}{2} \sigma_{x}^{2}}\right]^{2} \\
& =\alpha^{2} q^{2}-2 \alpha^{2} q^{2} e^{\mu_{x}+\frac{1}{2} \sigma_{x}^{2}}+\alpha^{2} q^{2} e^{2 \mu_{x}+2 \sigma_{x}^{2}}-\left[\alpha q-\alpha q e^{\mu_{x}+\frac{1}{2} \sigma_{x}^{2}}\right]^{2}
\end{aligned}
$$


So that $E\left[e^{\alpha V_{t}\left(1-e^{x}\right)}\right]$ is now

$$
e^{\alpha q-\alpha q e^{\mu x+\frac{1}{2} \sigma_{x}^{2}}+\frac{1}{2}\left[\alpha^{2} q^{2}-2 \alpha^{2} q^{2} e^{\mu_{x}+\frac{1}{2} \sigma_{x}^{2}}+\alpha^{2} q^{2} e^{2 \mu_{x}+2 \sigma_{x}^{2}}-\left[\alpha q-\alpha q e^{\mu_{x}+\frac{1}{2} \sigma_{x}^{2}}\right]^{2}\right]}
$$

Therefore our rare-event premium is,

$$
\lambda E\left(e^{x}\right)-\lambda q E\left(e^{x+\alpha V_{t}\left(1-e^{x}\right)}\right)-\lambda+\lambda q E\left(e^{\alpha V_{t}\left(1-e^{x}\right)}\right)
$$

which is just the rare-event premium of Theorem 3.1 and implies that our equilibrium equity premium is

$$
\begin{gathered}
\phi=\rho X_{0}(t)-\rho+\alpha \delta^{2}+\lambda e^{\mu_{x}+\frac{1}{2} \sigma_{x}^{2}}-\lambda q \exp \left[\mu_{x}+q \alpha-q \alpha e^{\mu_{x}+\frac{1}{2} \sigma_{x}^{2}}\right] \\
{\left[\frac{\exp \left\{\frac{1}{2}\left[\sigma_{x}^{2}+\mu_{x}^{2}+2 \alpha q \mu_{x}-2 \alpha q \mu_{x} e^{\mu_{x}+\frac{1}{2} \sigma_{x}^{2}}+\alpha^{2} q^{2}-2 \alpha^{2} q^{2} e^{\mu_{x}+\frac{1}{2} \sigma_{x}^{2}}+\alpha^{2} q^{2} e^{2 \mu_{x}+2 \sigma_{x}^{2}}\right]\right\}}{\exp \left\{\frac{1}{2}\left[\mu_{x}+\alpha q-\alpha q e^{\mu_{x}+\frac{1}{2} \sigma_{x}^{2}}\right]^{2}\right\}}\right]} \\
-\lambda+\lambda q e^{\alpha q-\alpha q e^{\mu_{x}+\frac{1}{2} \sigma_{x}^{2}}+\frac{1}{2}\left[\alpha^{2} q^{2}-2 \alpha^{2} q^{2} e^{\mu_{x}+\frac{1}{2} \sigma_{x}^{2}}+\alpha^{2} q^{2} e^{2 \mu_{x}+2 \sigma_{x}^{2}}-\left[\alpha q-\alpha q e^{\left.\left.\mu_{x}+\frac{1}{2} \sigma_{x}^{2}\right]^{2}\right]}\right.\right.}
\end{gathered}
$$

Theorem 4 An investor's equilibrium equity premium with quadratic utility function $U\left(r_{t}\right)=r_{t}-a r_{t}^{2}, a>0$ in the semimartingale market with normal jumps is given by

$$
\begin{gathered}
\phi=\rho X_{0}(t)-\rho+\frac{2 a \delta^{2}}{1-2 a V_{t}}+\lambda e^{\mu_{x}+\frac{1}{2} \sigma_{x}^{2}}-\frac{\lambda q e^{\mu_{x}+\frac{1}{2} \sigma_{x}^{2}}}{1-2 a q}+\frac{2 \lambda a q^{2} e^{2 \mu_{x}+2 \sigma_{x}^{2}}}{1-2 a q}-\lambda+\frac{\lambda q}{1-2 a q} \\
-\frac{2 \lambda a q^{2} e^{\mu_{x}+\frac{1}{2} \sigma_{x}^{2}}}{1-2 a q}
\end{gathered}
$$

where $\phi_{\delta}=\rho X_{0}(t)-\rho+\frac{2 a \delta^{2}}{1-2 a V_{t}}$ is the diffusive risk premium and $\phi_{N}=\lambda e^{\mu_{x}+\frac{1}{2} \sigma_{x}^{2}}-\frac{\lambda q e^{\mu_{x}+\frac{1}{2} \sigma_{x}^{2}}}{1-2 a q}+\frac{2 \lambda a q^{2} e^{2 \mu_{x}+2 \sigma_{x}^{2}}}{1-2 a q}-\lambda+\frac{\lambda q}{1-2 a q}-\frac{2 \lambda a q^{2} e^{\mu_{x}+\frac{1}{2} \sigma_{x}^{2}}}{1-2 a q}$ is the rare-event premium.

Proof. For the HARA Quadratic utility function,

$$
\phi_{N}=\lambda E\left[\left(e^{x}-1\right)\left(1-\frac{V_{t}\left(1-2 a V_{t} e^{x}\right)}{1-2 a V_{t}}\right)\right]
$$

and so

$$
\begin{aligned}
\phi_{N} & =\lambda E\left[e^{x}-\frac{V_{t} e^{x}-2 a V_{t}^{2} e^{2 x}}{1-2 a V_{t}}-1+\frac{V_{t}}{1-2 a V_{t}}-\frac{2 a V_{t}^{2} e^{x}}{1-2 a V_{t}}\right] \\
& =\lambda E\left[e^{x}-\frac{V_{t} e^{x}}{1-2 a V_{t}}+\frac{2 a V_{t}^{2} e^{2 x}}{1-2 a V_{t}}-1+\frac{V_{t}}{1-2 a V_{t}}-\frac{2 a V_{t}^{2} e^{x}}{1-2 a V_{t}}\right] \\
& =\lambda\left[E\left(e^{x}\right)-\frac{q E\left(e^{x}\right)}{1-2 a q}+\frac{2 a q^{2} E\left(e^{2 x}\right)}{1-2 a q}-1+\frac{q}{1-2 a q}-\frac{2 a q^{2} E\left(e^{x}\right)}{1-2 a q}\right]
\end{aligned}
$$

Now since $X \sim N\left(\mu_{x}, \sigma_{x}^{2}\right)$, we have that

$$
E\left(e^{x}\right)=e^{\mu_{x}+\frac{1}{2} \sigma_{x}^{2}}
$$

and

$$
E\left(e^{2 x}\right)=e^{2 \mu_{x}+2 \sigma_{x}^{2}}
$$

thus

$$
\phi_{N}=\lambda e^{\mu_{x}+\frac{1}{2} \sigma_{x}^{2}}-\frac{\lambda q e^{\mu_{x}+\frac{1}{2} \sigma_{x}^{2}}}{1-2 a q}+\frac{2 \lambda a q^{2} e^{2 \mu_{x}+2 \sigma_{x}^{2}}}{1-2 a q}-\lambda+\frac{\lambda q}{1-2 a q}-\frac{2 \lambda a q^{2} e^{\mu_{x}+\frac{1}{2} \sigma_{x}^{2}}}{1-2 a q}
$$

So that our equilibrium equity premium is now

$$
\phi=\rho X_{0}(t)-\rho+\frac{2 a \delta^{2}}{1-2 a V_{t}}+\lambda e^{\mu_{x}+\frac{1}{2} \sigma_{x}^{2}}-\frac{\lambda q e^{\mu_{x}+\frac{1}{2} \sigma_{x}^{2}}}{1-2 a q}+\frac{2 \lambda a q^{2} e^{2 \mu_{x}+2 \sigma_{x}^{2}}}{1-2 a q}-\lambda
$$




$$
+\frac{\lambda q}{1-2 a q}-\frac{2 \lambda a q^{2} e^{\mu_{x}+\frac{1}{2} \sigma_{x}^{2}}}{1-2 a q}
$$

We can conclude based on Figure 5 that the wealth value and equilibrium equity premium for the quadratic utility function are inversely proportional. Infact, the premium is zero whenever the wealth process becomes zero. All in all, the equilibrium equity premium for normally distributed jump amplitudes in the normalized semi martingale market does not differ significantly from the martingale market. However, the premium is highly dependent on the utility function of the investor.

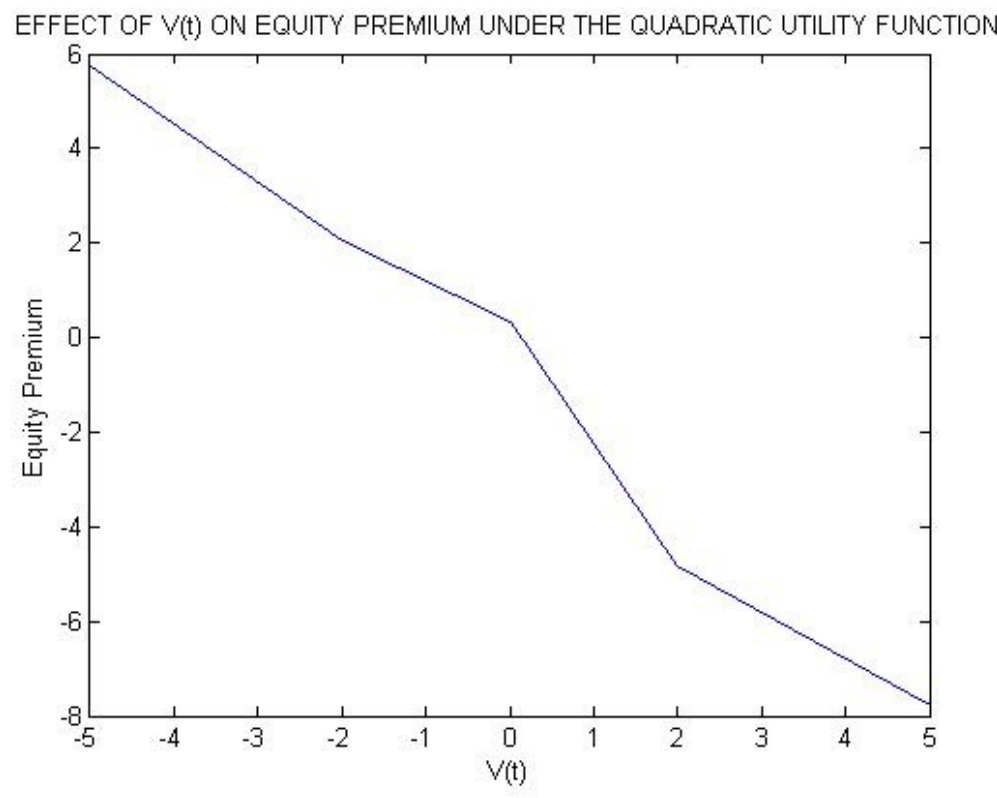

Figure 5. Quadratic Utility Wealth Effect

\section{Conclusion}

In the semimartingale market, the equilibrium equity premium for risk seeking investors is very attractive compared to that of risk averse investors in both martingale and semimartingale markets. This investor's premium is only affected by the wealth process in the rare-event premia. In addition, there is no optimal consumption for this investor in this market. The equity premium is almost zero whenever volatility is zero. It is also symmetrical about zero volatility. The mean and variance in the normality of jump sizes are directly proportional to the compensation received for having taken up some diffusive and jump risks in this market.

\section{References}

Aswath, D. (2012). Equity Risk Premiums (ERP): Determinants, Estimation and Implications. Stern School of Business.

Bates, D. (2008). The Market for Crash Risk. Journal of Economic Dynamics and Control, (32). https://doi.org/10.1016/j.jedc.2007.09.020

Bellamy, N., \& Jeanblanc, M. (2000). Incompleteness of markets driven by a mixed diffusion. Finance and Stochastics. https://doi.org/10.1007/s007800050012

Eberlein, E., \& Jacod, J. (1997). On the range of option prices. Finance and Stochastics. https://doi.org/10.1007/s007800050019

El Karoui. (1998). Robustness of the black and scholes formula. Mathematical Finance, 9(2). https://doi.org/10.1111/14679965.00047

Frey, R., \& Sin, C. A. (1999). Bounds on european option prices under stochastic volatility. Mathematical Finance, 9(2). https://doi.org/10.1111/1467-9965.00064

Frontczak, R. (2013). Pricing options in jump diffusion models using mellin transforms. Journal of Mathematical Finance. https://doi.org/10.4236/jmf.2013.33037

Gushchin, A., \& Mordecki, E. (2002). Bounds on option prices for semimartingale market models. Proc. Steklov Inst. 
Math.

Henderson, V. (2005). Analytical comparisons of option prices in stochastic volatility models. Mathematical Finance. https://doi.org/10.1111/j.0960-1627.2005.00210.x

Henderson, V. (2003). A comparison of q-optimal option prices in a stochastic volatility model with correlation. Oxford Financial Research Centre Preprint.

Henderson, V., \& Hobson, D. G. (2003). Coupling and option price comparisons in a jump diffusion model. Stochastics and Stochastic Rep. https://doi.org/10.1080/1045112031000084343

Hobson, D. G. (1998). Volatility misspecification, option pricing and superreplication via coupling. Ann. Appl. Probability. https://doi.org/10.1214/aoap/1027961040

Jakubenas, P. (2002). On option pricing in certain incomplete markets. Steklov Inst. Math.

Mukupa, G. M., \& Offen, E. R. (2015). The impact of utility functions on the equilibrium equity premium in a production economy with jump diffusion. IAENG International Journal of Applied Mathematics, 45(2).

Mukupa, G. M., \& Offen, E. R. (2016). Equity premium under normally distributed jump sizes in a production economy with jumps. International Journal of Applied Mathematics and Statistics, 54(2).

Mukupa, G. M., Offen, E. R., Kunda, D., \& Lungu, E. M. (2016). A comparative study of Equilibrium Equity Premium under Discrete Distributions of Jump Amplitudes. Mathematical Finance Journal, 6(1), 232-246.

Mukupa, G. M., Offen, E. R., \& Lungu, E. M. (2016). The Risk Averse Investors Equilibrium Equity Premium In A Semimartingale Market With Arbitrary Jumps. Mathematics Research Journal, 8(6), 139-147.

Mukupa, G. M., \& Offen, E. R. (2018a). Equilibrium Equity Premium in Semimartingale Market when Jump Amplitudes Follow a Binomial Distribution. Mathematical Finance Journal, 8(3), 599-612.

Mukupa, G. M., \& Offen, E. R. (2018b). The Semimartingale Equilibrium Equity Premium For Risk Neutral Investors. Financial Engineering Journal, 5(4). https://doi.org/10.1142/S2424786318500354

Møller, T. (2004). Stochastic orders in dynamic reinsurance markets. Finance and Stochastics. https://doi.org/10.1007/s00780-004-0130-y

Norstad, J. (2010). Introduction to porfolio Theory. Journal of Mathematical Economics, 20(1), 1-18.

Simon, G., \& John, Q. (2005). What Does the Equity Premium Mean? The Economics’ Voice.

Zhang, J. E., Zhao, H. M., \& Chang, E. C. (2012). Equilibrium Asset and Option Pricing Under Jump Diffusion. The University of Hong Kong. Mathematical Finance, 22. https://doi.org/10.1111/j.1467-9965.2010.00468.X

\section{Copyrights}

Copyright for this article is retained by the author(s), with first publication rights granted to the journal.

This is an open-access article distributed under the terms and conditions of the Creative Commons Attribution license (http://creativecommons.org/licenses/by/4.0/). 